\title{
Fiber Optic Fluorescence Temperature Monitoring System for Electric Apparatus
}

\author{
Lei Zhang ${ }^{1,}$ a , Wenqing Wang ${ }^{1}$, Weihua Zhang ${ }^{1}$, Xin Dai $^{1}$ \\ ${ }^{1}$ Shenyang Fire Research Institute, Shenyang, 110034, China \\ aemail: paul840112@hotmail.com
}

Keywords: Optical Fiber; Fluorescence; HV Apparatus; Temperature Monitoring

\begin{abstract}
Electric energy has become an important energy source in today's society. However, there has been a lack of safe, reliable and effective real-time fire monitoring solutions for high voltage switchgear, transformers and other critical electrical facilities in power plants and transformer substations. Therefore, the optical fiber fluorescence temperature sensor, which has the advantages of anti electromagnetic interference, small volume, high voltage resistance and corrosion resistance, has been developed. In this paper, the optical fluorescence sensor working principle and system structure are described, and technical targets are given by experimentation. The operating temperature range of fiber thermal probe is $-40^{\circ} \mathrm{C} \sim 200^{\circ} \mathrm{C}$, and the temperature resolution can reach $0.13^{\circ} \mathrm{C}$. A low-cost, safe and reliable detection solution is provided for the high-voltage electrical equipment fire monitoring.
\end{abstract}

\section{Introduction}

In today's society, the demand of electric power is increasing year by year. With the accelerated process of electrification, the hazards of electrical fire has attracted more and more attention. Thus far, the majority of electrical equipment has adopted closed structure, poor cooling effect and large heat accumulation, which easily leads to elevated temperature, insulation performance degradation, and equipment burning in some extreme cases. Hence, the real-time temperature monitoring of electrical equipment is critical to the safe operation of electrical equipment.

Traditional thermometer (such as thermocouple, thermal resistance) with electromagnetic properties of materials is susceptible to electromagnetic interference, so that the application has a high requirement on the environment [1]. In contrast, fiber temperature measurement technique has plenty of advantages, such as intrinsically safe, good stability, good electrical insulation, anti-electromagnetic interference, simple structure, small size, light weight, low price, etc [2]. Because the fluorescence lifetime does not depend on the fluorescence intensity, the fiber optical thermometer based on fluorescence lifetime measurement is completely unaffected by the degradation of light sources and detectors and the loss of fiber bending. In consequence, the optical fiber fluorescence thermometer as one of the most practical optical fiber temperature sensors is ideal for monitoring the temperature of electrical equipment [3-5].

This paper describes the principle and characteristic of fluorescence temperature measurement technology, and there is a linear relationship between fluorescence lifetime and temperature under the condition of $25^{\circ} \mathrm{C} \sim 200^{\circ} \mathrm{C}$, and the structure and characteristics of fluorescence thermo-sensor are reported in detail. 


\section{Principle}

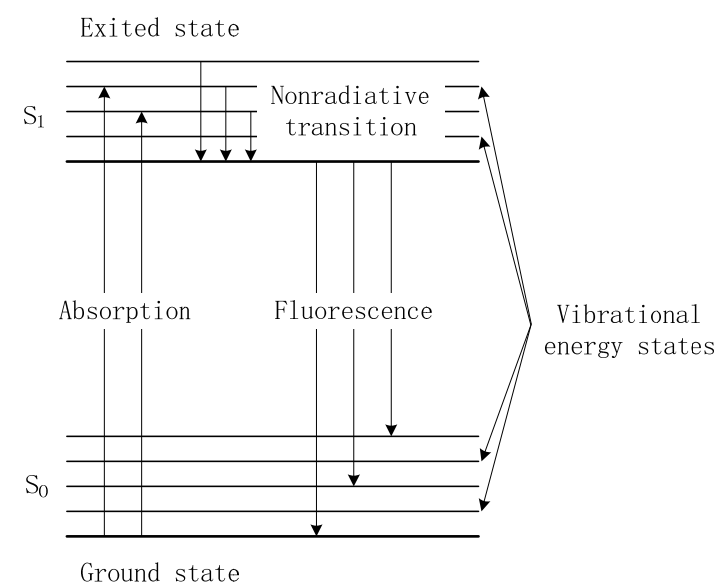

Fig. 1. Fluorescence generation principle

The fluorescence generation principle is shown in Fig.1. The orbital electrons of molecules can exist in two major states: the ground state (S0), and the excited state (S1). These two states are singlet states. These energy states can further be divided into smaller energy levels as a result of vibrational energy levels. When light energy is incident on certain molecules capable of fluorescence, the orbital electron systems of these molecules first absorb the incident light. The electrons in these systems therefore go from the ground state to the excited energy state (S1). Furthermore these electrons jump to different vibrational levels of the excited state as can be seen in the above Fig.1. The electrons then go to the lowest vibrational energy level of the excited state via nonradioactive transition. Since these molecules are capable of fluorescing, the electrons now jump from the lowest energy level of the excited to state, to different vibrational energy levels of the ground state via the emission of specific quantum of energy in the form of light. This light thus emitted results in fluorescence.

The fluorescence lifetime refers to the average time the molecule stays in its excited state before emitting a photon. Fluorescence typically follows exponential form:

$$
I=I_{0} \exp \left(-\frac{t}{\tau}\right)
$$

where $I$ is the fluorescence intensity at time t, $I_{0}$ is the initial fluorescence intensity and $\tau$ is the fluorescence lifetime. This is an instance of exponential decay. And it is proved that the fluorescence lifetime as a function of temperature, is independent of the fluorescence intensity. Therefore, the sensitivity and the accuracy of the measurement are only influenced by the value and the temperature coefficient of the fluorescence lifetime.

\section{System structure}

The temperature measurement system is shown in Fig. 2.

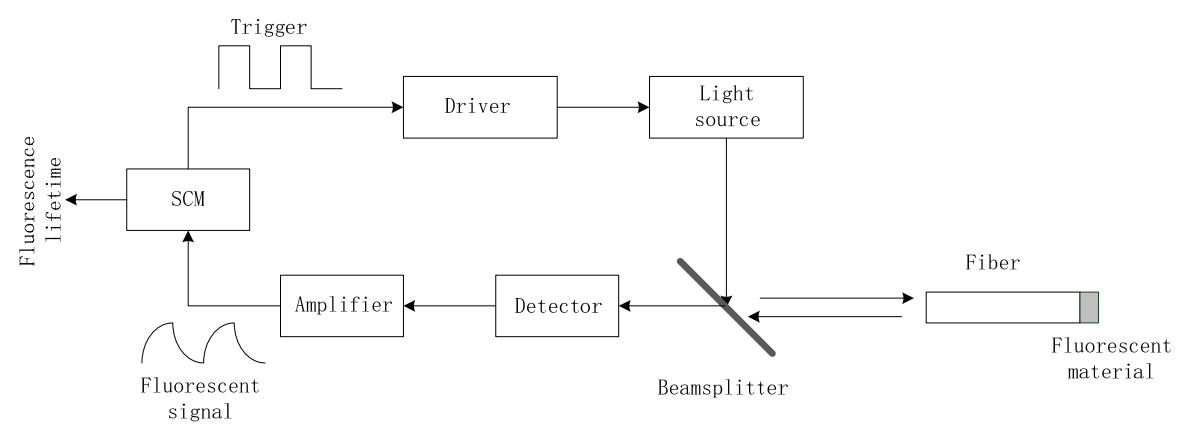

Fig. 2. System configuration diagram

The temperature monitoring system is composed of SCM, light system, optical splitting system, 
detection system and optical fiber fluorescence probe. The fluorescent material is glued on the optical fiber end face. When the UV light is coupled into the optical fiber, fluorescent material emits visible red light which's lifetime is related to the ambient temperature. The sensor head is in direct contact with the measured object, so as to realize the real-time temperature monitoring. The fiber fluorescence probe and other optoelectronic systems are connected by a conductive fiber, thereby achieving a good isolation of strong electromagnetic field and other harsh environment. Accordingly, the sensor has the characteristics of both high dielectric strength and anti electromagnetic interference.

The temperature measurement system works in a very simple way. First, the SCM sends out a control signal to trigger a pulse of light, and the UV light reflected by the beam splitter is coupled into the optical fiber. Then, the fluorescent material on the fiber tip excited by the optical pulse emits fluorescence signal carrying temperature information, and the signal amplitude decays with time. After that, the fluorescence signal transmits back through the fiber, and it is separated by the beam splitter and received by the photoelectric part. Subsequently, the weak current obtained by photoelectric conversion, through amplification system, is collected and processed using the method of least squares. Finally, the fluorescence lifetime $\tau$ is acquired.

\section{Experiment}

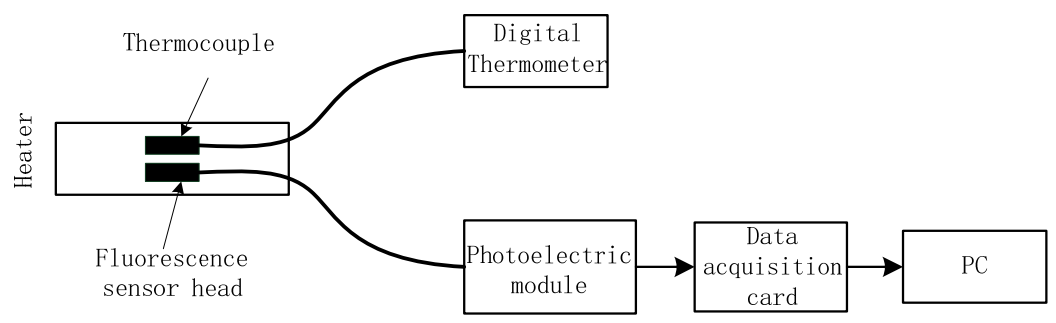

Fig. 3. Fluorescence temperature sensor experimental system

The experimental system is shown in Fig. 3. Optical fiber sensor head and the thermocouple sensor are fixed together on the heating plate to ensure that they feel the same temperature. The fluorescence signal is transmitted to the photoelectric module via optical fiber, and transformed to an analog signal. The analog signal collected by the data acquisition card converts to the digital signal which is transmitted to the computer to display. Data acquisition card collects and transfers the results to a computer displaying output. The exponential decay of fluorescence signal collected by high-speed A/D conversion apparatus is shown in Fig. 4.

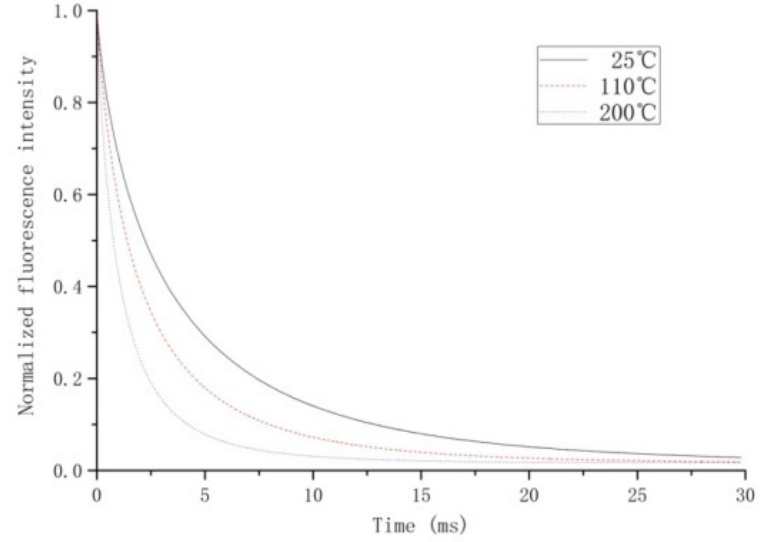

Fig. 4. Fluorescence decay curves

The figure shows the normalized attenuation curves of fluorescence signal at different temperatures. Obviously, the decay rate of fluorescence signal is associated with the temperature, the higher the temperature, the faster the signal attenuation.

The fluorescence lifetime can be calculated by performing least squares fitting on the acquired signals. The fluorescence probe is placed in a thermostatic oven, and Fig. 5 shows the temperature-fluorescence lifetime calibration curve. 


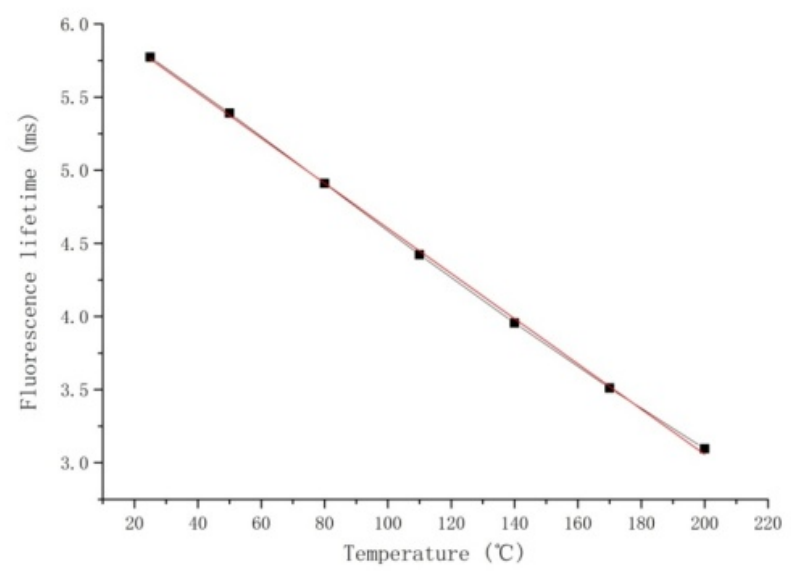

Fig. 5. The temperature dependence of the fluorescence lifetime

As shown above, the temperature is increased from $25^{\circ} \mathrm{C}$ to $200^{\circ} \mathrm{C}$, simultaneously fluorescence lifetime is decreased from $5.773 \mathrm{~ms}$ to $3.096 \mathrm{~ms}$. The temperature sensitivity is about $0.015 \mathrm{~ms} /{ }^{\circ} \mathrm{C}$. As can be seen from the calibration results, there is a good linear relationship between fluorescence lifetime and temperature, and the linear fitness of the measured data has reached 0.9994 .

In order to get the temperature resolution, the sensor head is arranged at a indoor temperature of $22.9^{\circ} \mathrm{C}$, and continuously monitored for $300 \mathrm{~s}$. The measurement curve is shown in Fig.6. The fluctuation of the fluorescence lifetime is less than $0.002 \mathrm{~ms}$ during $300 \mathrm{~s}$. According to temperature sensitivity, the temperature resolution is about $0.13^{\circ} \mathrm{C}$.

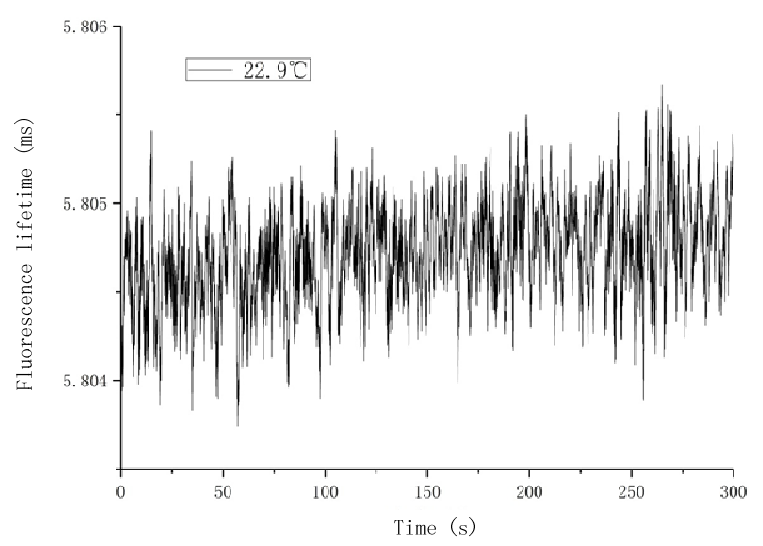

Fig. 6. Continuous measurement for $300 \mathrm{~s}$

\section{Conclusion}

Under the influence of high voltage, microwave, strong electromagnetic interference and other harsh environment, traditional temperature measurement instruments are vulnerable to interference, and they are difficult to effectively ensure the safe operation of power facilities. In this paper, fluorescence optical fiber temperature sensor was developed with the advantages of simple structure, fast response and good stability, and installed easily and flexibly. The sensor head and transmission fiber are high temperature resistant, corrosion resistant and easy to bend. Accordingly, the device is suitable for working in small space, high voltage, and high magnetic field environments. Therefore, the temperature sensor designed in this paper provides a new and relatively inexpensive way of temperature measurement for high voltage electrical equipment temperature monitoring, and the successful development has great practical significance. 


\section{References}

[1] David Monzón-Hernández, Donato Luna-Moreno, Dalia Martínez-Escobar. Fast response fiber optic hydrogen sensor based on palladium and gold nano-layers. Sensors and Actuators B: Chemical, 2009, 136(2): 562-566.

[2] Babnik A, Kobe A. Improved probe geometry for fluorescence-based fiber-optic temperature sensor[J]. Sensors and Actuators, 1996(57): 203-207.

[3] Fen Ying, Huang Shihua. The analysis of sinusoidal modulated method used for measuring fluorescence lifetime. Spectrosc. Spectral Anal. , 2007, 27(12): 2523-2526

[4] Vincent Minier, J. L. Lovato, Dominique Persegol. Thermal diagnosis of medium voltage switchboard: a cost effective solution based on a multipoint optical sensor. SPIE, 2000, 4074: 184-192.

[5] M Stanciu, K.T.V. Grattan. LiCAF crystal based optical fiber thermometry. Sensors and Actuators A, 2002, 99: 277-283. 